\title{
Novel external fixation fracture method with circular locking mechanism compared with the application of dynamic axial external fixator on experimental tibial model ensures better stability in bending and favourable performance in dynamic loads
}

\author{
Arsen Pavica,*, Janos Kodvanj ${ }^{\mathrm{b}}$, Srecko Sabalic', Fabijan Cukelja, Bore Bakota ${ }^{\mathrm{d}}$ \\ ${ }^{a}$ University Hospital Centre Split, Surgery Clinic, Department of Traumatology, Split, Croatia \\ ${ }^{b}$ Faculty of Mechanical Engineering and Naval Architecture, University of Zagreb, Zagreb, Croatia \\ "University Hospital Centre "Sisters of Mercy", Clinic for Traumatology, Zagreb, Croatia \\ ${ }^{d}$ General Hospital Karlovac, Department of Traumatology, Karlovac, Croatia
}

\section{K E Y W O R D}

\section{Tibial fracture}

External fixator

Novel external fixator

Dynamic axial external fixator

Dynamic load

Static load

Cyclic load

Biomechanics

Croatia

\begin{abstract}
A B S T R A C T
Objective: The aim of this study was to compare the biomechanical properties of a novel tibial external bone fracture fixator with a circular locking mechanism with standard dynamic axial external fixator. Material and methods: In order to investigate the prototype usability in experimental conditions, a biomechanical study was performed in which 42 polyacetal tubes set in 14 experimental groups and subgroups represented the fractured tibia that were fixed by a standard dynamic axial external fixator and a novel fixator. Displacements under static and dynamic loads were measured, with static ones corresponding to three directions of fragment movement and dynamic simulating the human gait. Analysis was performed in SPSS v13, with significance set at $\mathrm{P}<0.05$.

Results: The novel fixator showed biomechanical superiority in "fragments apart" study groups, while the standard dynamic axial external fixator outperformed the novel one in the situations of bending with "fragments in contact" study groups. There were no significant differences in dynamic load, despite better numerical result of the novel fixator.

Conclusion: The novel fixator is expectedly faster applicable and offers greater extent of external fixation flexibility. Further developments of this model thus seems justified in both construction improvement and on clinical application.
\end{abstract}

(c) 2013 Elsevier Ltd. All rights reserved.

\section{Introduction}

External fixation is a process of bone fragment fixation using the elements that rely on external mechanical construction, based on three basic approaches: the pins and wires should avoid damage to vital structures, allow access to the area of injury, and should meet the mechanical demands of the patient and the injury. ${ }^{1,2}$ The use of external fixation for tibial fractures became widely accepted over the last 30 years. ${ }^{3,4}$ However, various approaches that are used are also linked to some limitations, including technical requirements and complexity of fixator application, possibility for misalignment, exposure to radiation and they are often described as non-patient friendly. ${ }^{5,6}$ A recent overview of different methods of external fixation suggested that there is an insufficient amount of evidence that would show that any of the approaches should be favoured ${ }^{7}$, suggesting that there is a room for further improvements that could reduce these

\footnotetext{
* Corresponding author at: Clinical Hospital Centar Split, Spinciceva 5, 21000 Split, Croatia. Tel.: +385981956956; Fax: +38521557300.

E-mail address: arsen.pavic@gmail.com (A. Pavic).
}

limitations. Therefore, the aim of this study was to investigate the basic biomechanical properties of a novel tibial fracture fixator with circular locking mechanism, designed for faster application and greater extent of fixator flexibility.

\section{Materials and methods}

For this study, a novel prototype of an external tibial fixator was constructed and tested. The basic construction requirements for the fixator were to allow greater flexibility (by providing greater angles and mobility of fixator elements), to reduce the time needed for its surgical application and to reduce the need for pins repositioning. These requirements were met with the development of a circulatory locking mechanism, which is locked by a "butterfly" lever (Figure1). The prototype of the novel fixator was produced from the ISO 5832-1 steel.

Biomechanical properties of the constructed novel fixator were compared to a standard dynamic axial external fixator (Orthofix ${ }^{\circledR}$ SLR, Verona, Italy) in an experimental study design.

Polyacetal models $(n=42)$ simulating tibia were used $(30 \mathrm{~mm}$ in diameter each and $200 \mathrm{~mm}$ in length each) and fixed with six 


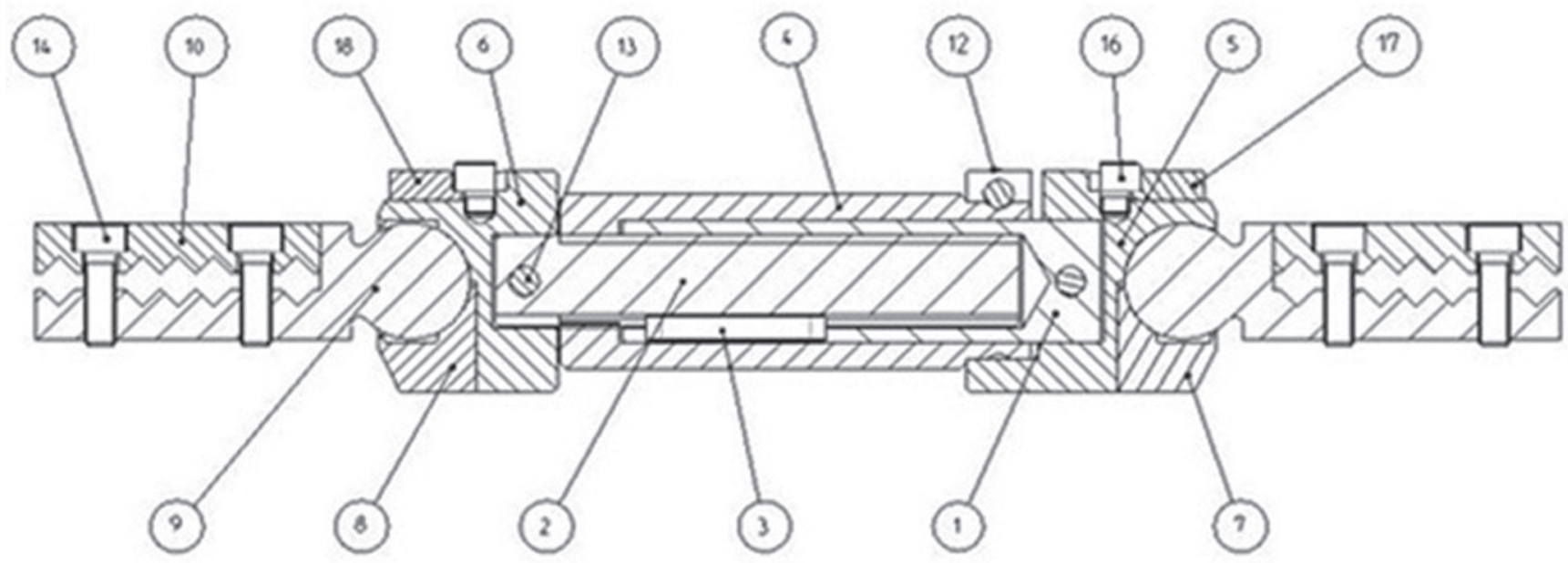

Fig. 1. Schematic cross section of the novel fixator prototype. Elements: 1 fixed rod, 2 moving rod, 3 feather, 4 connecting arm, 5 left joint, 6 right joint, 7 lower left joint, 8 lower right joint, 9 ball, 10 pin holder, 12 arm screw, 13 rod screw, 14 pin screw, 16 securing lever screw, 17 left lever, 18 right lever.

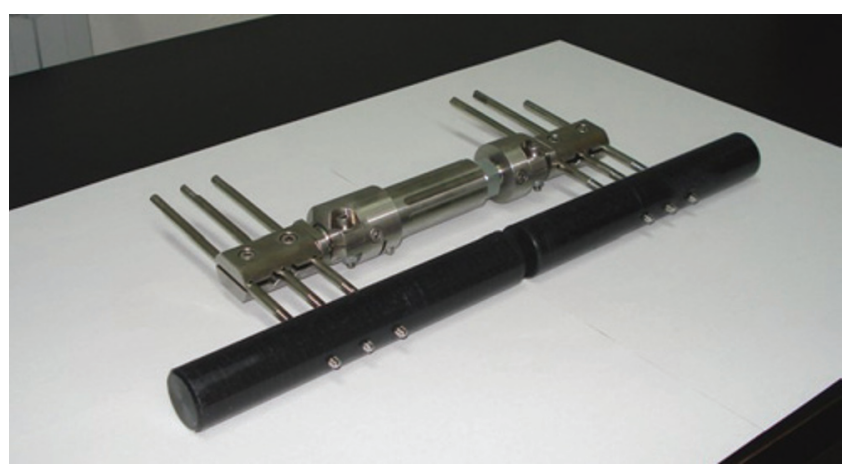

Fig. 2. Model of a novel external fixation fracture method with fragments apart.

Table 1

Fixated tube fragment movements in $\mathrm{mm}$ - results from the experimental measurements

\begin{tabular}{lccc}
\hline $\begin{array}{l}\text { Measurement (mm); } \\
\text { mean } \pm \text { standard deviation }\end{array}$ & $\begin{array}{c}\text { Novel } \\
\text { fixator }\end{array}$ & $\begin{array}{c}\text { Ortofix } \\
\text { fixator }\end{array}$ & $\begin{array}{c}\mathrm{P} \\
\text { (t-test) }\end{array}$ \\
\hline $\begin{array}{l}\text { Longitudinal movement, bending (y-axis) } \\
\quad \text { Fragments in contact }\end{array}$ & $0.91 \pm 0.01$ & $0.52 \pm 0.03$ & $<0.001$ \\
$\quad 0.85 \pm 0.04$ & $1.32 \pm 0.03$ & $<0.001$ \\
$\quad$ Fragments apart & $0.03 \pm 0.01$ & $0.02 \pm 0.01$ & 0.006 \\
Lateral movement, bending (x-axis) & $0.08 \pm 0.01$ & $0.81 \pm 0.11$ & $<0.001$ \\
$\quad$ Fragments in contact & $0.10 \pm 0.01$ & $0.09 \pm 0.02$ & 0.041 \\
$\quad$ Fragments apart & $0.02 \pm 0.00$ & $0.06 \pm 0.01$ & 0.006 \\
Forward movement, bending (z-axis) & $0.78 \pm 0.26$ & $0.92 \pm 0.05$ & 0.447 \\
$\quad$ Fragments in contact & & & \\
$\quad$ Fragments apart & & & \\
Cyclic loads - Fragments apart & &
\end{tabular}

pins ( $6 \mathrm{~mm}$ in diameter each), three at each side of the created fracture. ${ }^{8}$ The space between the most inner pins was $186 \mathrm{~mm}$, and the distance between the bone models and the fixator was $40 \mathrm{~mm}$ (Figure 2).

Both types of fixators were placed on the bones (polyacetal models) in the same manner and had the same above mentioned characteristics.

Seven groups and subgroups to test were created for each fixator type, with three bones (polyacetal models) for measurements in each group (Table 1 ).

Two distinct situations were simulated: bone fragments in contact and bone fragments without contact - spaced $10 \mathrm{~mm}$ apart (Table 1). Also, two sets of displacement measurements were made; under static and under the dynamic load (Figure 3). The resulting bone fragments displacements were measured in three dimensions ( $\mathrm{x}, \mathrm{y}$ and $\mathrm{z}$ ), using a screw-drive testing

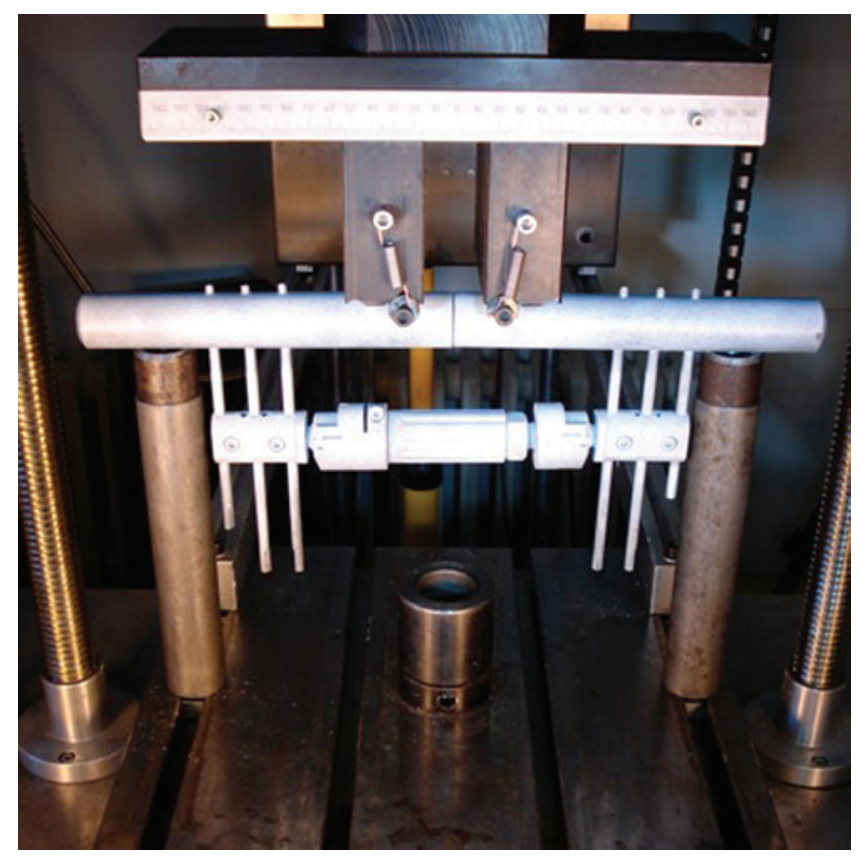

Fig. 3. Dynamic load model- Fragments in contact.

machine Messphysik BETA 50-5 (Messphysik, Austria; Figure 4). The bending tests were conducted with a maximum load of $250 \mathrm{~N}$. In all tests the loading and unloading speed was $5 \mathrm{~N} / \mathrm{s}$. Dynamic tests were carried out in an asymmetrical fashion, using a servohydraulic testing machine LFV-50-HH (Walter Bai, Switzerland; Figure 5), with DIGWIN 2000-EDC120 digital control system. Cyclic tests were perfomed with a sinusoidal loading between 0 and $200 \mathrm{~N}$ in a force control at $1 \mathrm{~Hz}$ for 10,000 cycles. This type of testing simulated human gait (Figure6).

In the static tests all displacements were determined using the non-contact 3D optical measuring system Aramis 4M (GOM, Germany; Figure 7), with two digital CCD Dalsa Falcon 4M60 cameras, two Titanar lenses, framegrabbers X64CL iPro and Aramis software v6.2. Measurements were made to correspond to fragment displacement in $\mathrm{y}, \mathrm{x}$ and $\mathrm{z}$ axis. In the cyclic tests the displacements were recorded with the machine's own software (DIONPro+ ver. 4.58). Statistical analysis was based on means and standard deviation calculation, followed by the use of t-test. Analysis was performed in SPSS v13 (SPSS Inc, Chicago, IL), with significance set at $\mathrm{P}<0.05$. 


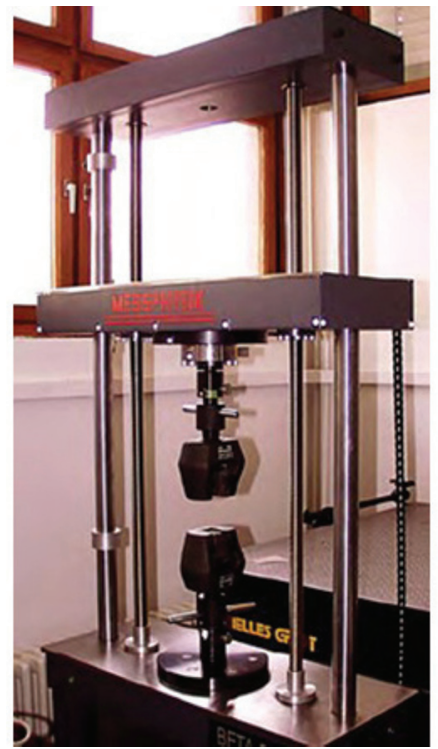

Fig. 4. Screw-drive testing machine Messphysik BETA 50-5.

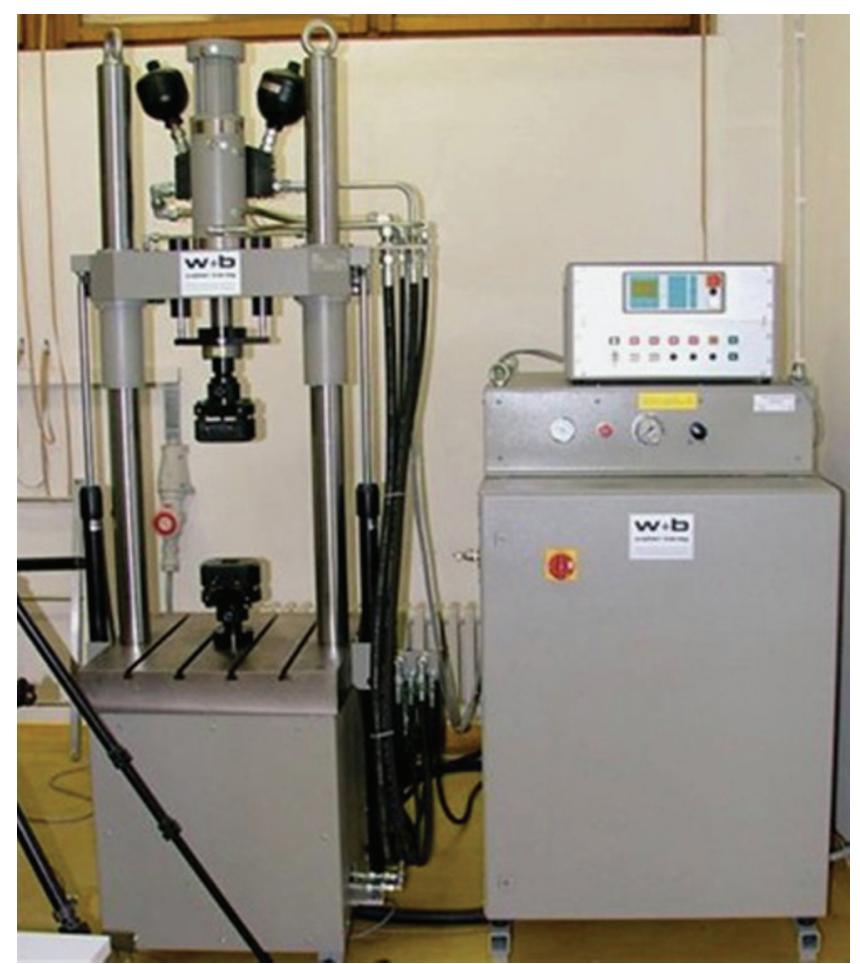

Fig. 5. Servo-hydraulic testing machine LFV-50-HH.

\section{Results}

The results of the measurements in all of the fourteen experimental groups and subgroups indicated a fair share of statistically significant differences (Table 1 ).

The dynamic axial external fixator outperformed the novel one in the situations of bending with simulated bone fragment contact (Table 1). Conversely, the novel fixator outperformed the dynamic axial external fixator in situations of bending with a simulated bone loss and a distance between the bone fragments (Table 1). Lastly, no significant difference was seen in dynamics loads, despite better numerical result of the novel fixator (Table 1).

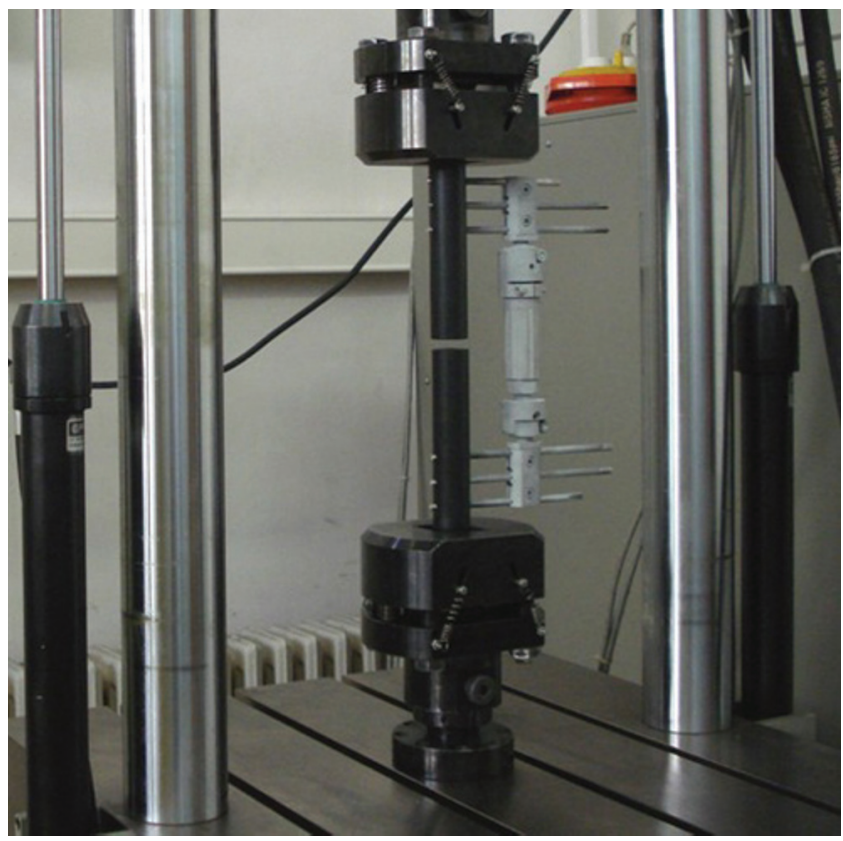

Fig. 6. Cyclic load model- Fragments apart.

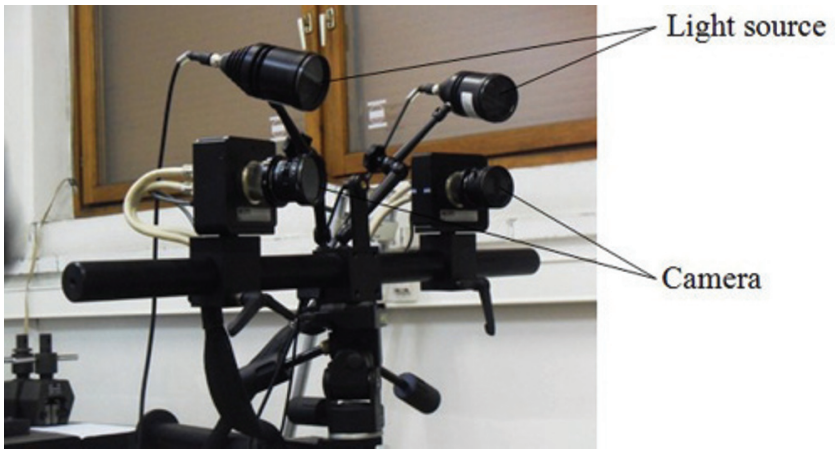

Fig. 7. Non-contact 3D optical measuring system Aramis 4M.

\section{Discussion}

These results suggest that novel tibial fixator with circulatory locking mechanism may prove beneficial in situations when in multifragmentary bone trauma bending forces are involved. Furthermore, dynamic load analysis yielded lesser fragment movement in a novel fixator, despite the lack of formal statistical significance. However, novel fixator was developed in order to allow the greater application flexibility (ensured with greater angle extent and butterfly locking which allows faster and easier post-operative management), simpler application which reduces fixation time, reduced probability for pins re-repositioning and thus greater overall flexibility. These properties make it an interesting tool not only for selected tibial fractures in trauma surgery, but also for wartime casualties, where speed and flexibility may outweigh over the other fixator models. Based on these properties and the results of this study, two developmental directions will be pursued. The first one includes further prototype development, aimed at the use of novel materials (titanium and carbon fibres) and additional construction improvements. The second one will be based on extension of the indication, aiming for the application in metaphyseal tibial fractures or distal femur fractures. These improvements are likely to at least reduce some of the problems related to the external fixator application., 5

This study suffers from several limitations, ranging from the fact that model was represented by a polyacetal tube ${ }^{8}$ and that 
only a limited number of measurements were made for the static loads. Furthermore, the experiment did not take into account other structures and wound properties, thus producing a set of rather limited, but promising results that all need to be validated in live tissue before further steps towards product development and wider use in humans is possible. Analysis of the application time seems favourable, but this should also be tested on the clinical setting before a more general conclusion can be made. Nevertheless, the potential benefits of this approach are promising, thus supporting the long and windy road to the commercial product development.

In conclusion, the novel fixator is expectedly quicker applicable and offers greater extent of external fixation flexibility. Further developments of this model thus seem justified in both construction improvement and on clinical application.

\section{Conflict of interest statement.}

Authors declare no conflict of interest.

\section{Acknowledgements.}

The prototype development has been funded by the company Instrumentaria from Zagreb, Croatia.

\section{References}

1. Behrens F. General theory and principles of external fixation. Clin Orthop Relat Res 1989;241:15-23.

2. Fragomen AT, Rozbruch SR. The mechanics of external fixation. HSS J 2007;3:13-29.

3. Bach AW, Hansen ST Jr. Plates versus external fixation in severe open tibial shaft fractures - a randomized trial. Clin Orthop Relat Res 1989;241:89-94.

4. Kenwright J, Gardner T. Mechanical influences on tibial fracture healing. Clin Orthop Relat Res 1998;355(Suppl 1):179-90.

5. El-Sayed M, Atef A. Management of simple (types A and B) closed tibial shaft fractures using percutaneous lag-screw fixation and Ilizarov external fixation in adults. Int Orthop 2012;36:2133-8.

6. Feng W, Fu L, Liu J, Qi X, Li D, Yang C. Biomechanical evaluation of various fixation methods for proximal extra-articular tibial fractures. J Surg Res 2012;178:722-7.

7. Hutchinson AJ, Frampton AE, Bhattacharya R. Operative fixation for complex tibial fractures. Ann R Coll Surg Engl 2012;94:34-8.

8. Ryan MD. Polyacetal rod fixation of fractures in osteoporotic bone. A preliminary report. J Bone Joint Surg Br 1991;73-B:506-8. 